\title{
Consenso em Reumatologia Pediátrica Parte I - Definição dos Critérios de Doença Inativa e Remissão em Artrite Idiopática Juvenil/Artrite Reumatóide Juvenil(*)
}

\author{
Consensus in Pediatric Rheumatology \\ Part I - Criteria Definition of Inactive Disease and Remission in Juvenile \\ Idiopathic Arthritis / Juvenile Rheumatoid Arthritis
}

\author{
Claudia Machado(1), Nicolino Ruperto ${ }^{(2)}$
}

\begin{abstract}
RESUMO
Não há critérios universalmente aceitos para a remissão clínica em artrite idiopática juvenil/artrite reumatóide juvenil (AIJ/ARJ). Objetivo: formar consenso sobre estes critérios. Métodos: foi utilizado um inquérito pelo método Delphi para reunir os critérios vigentes e utilizados por especialistas em reumatologia pediátrica (RP) no mundo todo. A análise dos resultados constituiu a base para uma Consensus Conference utilizando a nominal group technique (NGT) para alcançar o consenso nas questões não resolvidas após a análise dos questionários deste inquérito. Cento e trinta RP de 34 países responderam ao inquérito e 20 RP de nove países elegeram os critérios durante dois dias, em processo de discussão estruturada, para formar consenso pela NGT. Resultados: os critérios de doença inativa deveriam incluir: 1) nenhuma articulação com artrite em atividade; 2) ausência de febre, rash, serosite, esplenomegalia ou linfadenopatia generalizada atribuída à AIJ/ARJ; 3) ausência de uveíte em atividade; 4) VHS ou PCR negativas (se ambos forem testados, ambos devem ser normais); 5) a avaliação global pelo médico deve indicar o melhor escore possível, indicando doença inativa. Conclusões: de acordo com o voto de consenso, seis meses contínuos de doença inativa são necessários para se considerar um paciente em estado de remissão com medicação; 12 meses contínuos de doença inativa e sem medicação são necessários para considerar um paciente em estado de remissão sem medicação. O critério para remissão sem medicação deve prever com acurácia de 95\% a probabilidade inferior a $20 \%$ de recaída em cinco anos.
\end{abstract}

Palavras-chave: artrite idiopática juvenil, artrite reumatóide juvenil, consenso, remissão.

\begin{abstract}
Validated and widely accepted criteria for clinical remission in JIA/JRA do not currently exist. Objective: To achieve consensus in this matter. Methods: The Delphi consensus-formation approach was used to gather the criteria in use by pediatric rheumatologists (PR) worldwide. Results from the questionnaires provided the basis for the development of a consensus conference using the nominal group technique (NGT) to reach consensus on questions not solvable by the questionnaire format. One hundred and thirty PR from 34 countries responded the Delphi questionnaires and 20 PR from 9 countries attended a 2-day consensus conference. Results: Consensus results were: criteria for inactive disease should include: 1) no active arthritis; 2) no fever, rash, serositis, splenomegaly, or generalised lymphadenopathy attributable to JIA/JRA; 3) no active uveitis; 4) normal ESR or CRP (if both are tested, both must be normal); 5) a physician's global assessment of disease activity rated at the best score possible and indicating no disease activity. Conclusions: According to consensus vote, 6 continuous months of inactive disease are necessary before classifying a patient as in remission on medication; 12 months off medication while maintaining inactive disease are necessary to classify a patient as in remission off medication. The criteria for in remission off medications should predict with 95\% accuracy that a patient has a $20 \%$ probability of disease relapse within the next 5 years.
\end{abstract}

Keywords: consensus, juvenile idiopathic arthritis, juvenile rheumatoid arthritis, remission.

\footnotetext{
Trabalho realizado na Disciplina de Reumatologia Pediátrica do Departamento de Pediatria da Faculdade de Medicina de Botucatu da Universidade Estadual Paulista (Unesp), Botucatu-SP, Brasil. Recebido em 22/07/2004. Aprovado, após revisão, em 09/12/2004.

1. Professora Assistente Doutora. Disciplina de Reumatologia Pediátrica do Departamento de Pediatria da Faculdade de Medicina de Botucatu da Unesp, Botucatu-SP, Brasil. Coordenadora e Representante Nacional do Paediatric Rheumatology International Trials Organization (Printo).

2. Senior Scientist. Paediatric Rheumatology International Trials Organization - (Printo) e Instituto G. Gaslini, Gênova, Itália.
}

Endereço para correspondência: Claudia Machado. Departamento de Pediatria. Faculdade de Medicina de Botucatu - Campus da Unesp, CEP 18618-970, Rubião Junior, Botucatu, SP, Brasil. Telefone: (14) 3811-6274 / (14) 3811-6083, fax: (14) 3882-0421. E-mail: cmachado@fmb.unesp.br 


\section{INTRODUÇÃO}

Nas últimas décadas houve grande progresso no tratamento das artrites juvenis. O reconhecimento precoce de suas complicações, como a síndrome de ativação macrofágica, principalmente na forma sistêmica, resultou em declínio na mortalidade. A intervenção mais precoce nas formas poliarticulares também tem levado à evolução com menor incapacidade física e psicossocial. Observou-se também melhora no prognóstico visual, com a vigilância oftalmológica regular mediante o risco de uveíte crônica. Isto se deve principalmente ao aprimoramento de técnicas diagnósticas, laboratoriais e por imagem e à introdução de novos agentes terapêuticos, como as drogas anti-reumáticas e os agentes biológicos. Contudo, a mortalidade ainda é um risco, especialmente para os pacientes com persistência da sinovite ativa na vida adulta ${ }^{(1)}$. Até mesmo durante a infância há aumento da mortalidade de crianças com artrite reumatóide juvenil (ARJ)/artrite idiopática juvenil (AIJ) com relação a seus pares ${ }^{(2)}$.

A avaliação do prognóstico a longo prazo da ARJ/AIJ indica a persistência de atividade da doença durante a adolescência e vida adulta numa grande proporção de pacientes, com repercussões clínicas e funcionais significantes ${ }^{(2-7)}$. Estima-se que 50\% das crianças com ARJ/AIJ terão doença recorrente ou persistente na vida adulta, resultando em destruição articular progressiva. As formas poliarticulares respondem por cerca de $40 \%$ de todos os casos e têm o pior prognóstico, com a probabilidade de remissão de apenas 15\% em dez anos. Conseqüentemente, a sinovite persistente poderá resultar em lesão articular com diminuição do desempenho nas atividades cotidianas, retardo motor de grandes e pequenas articulações, o que irá interferir no desempenho escolar e na qualidade de vida.

Paralelamente às novas estratégias terapêuticas atualmente aceitas para a artrite reumatóide no adulto, o paradigma do tratamento das artrites juvenis está evoluindo para a terapêutica mais precoce, também chamada mais "agressiva", e que tem por objetivo deter a progressão da atividade inflamatória antes que tenham ocorrido lesões ou dano articular permanente, e também para alcançar melhor desempenho funcional a longo prazo. A terapia precoce com o metotrexate, a terapia combinada envolvendo múltiplas medicações, e os agentes biológicos estão sendo cada vez mais utilizados em ARJ/AIJ, mesmo em indicações consideradas off label porque os ensaios clínicos randomizados para as artrites juvenis estão ainda em seus primeiros passos.
Como a resolução completa nas formas poliarticulares da ARJ/AIJ é incomum, para a maioria das crianças e adolescentes é administrado o o uso de múltiplas combinações medicamentosas durante muitos anos. Ainda não há estudo que identifique um tratamento que consistentemente alcance a resposta clínica completa, definida como a ausência de doença enquanto em uso de medicação, ou a remissão clínica. Porém, recentemente foram definidas por meio de consenso entre especialistas, as medidas essenciais de desfecho clínico e a definição de melhora clínica em ARJ, aplicáveis também para a definição mais abrangente das artrites crônicas juvenis - as artrites idiopáticas juvenis (AIJ). Esta definição foi obtida por inquérito e consenso entre especialistas, e teve a aprovação recente do American College of Rheumatology, sendo denominada ACR-30 $\operatorname{Ped}^{(8)}$ (acessível online em http://www.rheumatology.org).

Não há critérios válidos e universalmente aceitos como "critério de remissão" para a ARJ/AIJ; conseqüentemente, o termo é utilizado por clínicos e pesquisadores de maneira inconsistente na literatura. A padronização destes critérios proveria os profissionais da saúde e a pesquisa clínica com um instrumento para identificar e definir precisamente a situação clínica de um paciente e uma descrição mais acurada do desfecho clínico mediante a intervenção terapêutica, pois a expectativa é de que os novos tratamentos induzam à remissão.

Os objetivos deste projeto foram: desenvolver por meio de consenso entre especialistas os critérios de doença inativa, remissão em uso de medicação e remissão sem medicação, e decidir qual "status clínico" a definição deve prever, para que se dê ao termo a devida relevância clínica.

\section{CRITÉRIOS DE DOENÇA INATIVA E REMISSÃo EM ARJ/AIJ}

O objetivo deste projeto foi a promulgação da definição de inatividade e remissão para a ARJ/AIJ. Foi realizado sob o patrocínio do Paediatric Rheumatology International Trials Organization (PRINTO) em cooperação com o Paediatric Rheumatology Collaborative Study Group (PRCSG) - NIH-Children's Hospital of Cincinnati e Childhood Arthritis \& Rheumatology Research Alliance (CARRA), três organizações acadêmicas e grupos de trabalho multicêntricos e multinacionais voltados para a pesquisa clínica em reumatologia pediátrica.

Com o objetivo de buscar uma definição que seja válida e baseada em evidências, de um estado de "resposta clínica completa" ou controle da atividade da doença, ou "remissão clínica", foram realizados: 1. a busca sistemática de literatura; 
e 2. inquéritos entre especialistas em reumatologia pediátrica, voltados à prática do dia-a-dia e à prática acadêmica.

A primeira etapa deste trabalho foi realizada por meio de um inquérito postal, a Delphi Technique, e a segunda por meio da Consensus Conference - Nominal Group Technique ${ }^{(9)}$.

$\mathrm{O}$ inquérito postal foi enviado aos especialistas, contendo questões abertas e solicitando as variáveis a serem consideradas para a avaliação de atividade/inatividade da doença. O método Delphi é um método sistemático para a solicitação do julgamento clínico por meio de uma seqüência de questões, de tal forma que a primeira fase inclui questões abertas ou não estruturadas. Os questionários adicionais são construídos baseando-se nos resultados das respostas do anterior e as perguntas tornam-se seqüencialmente mais específicas à medida que se forma o Consenso. O método Delphi serve como trabalho preliminar para tornar as questões mais claras para a elaboração da discussão face-aface na Consensus Conference, onde almeja-se alcançar o consenso nas questões não resolvidas por meio de questionário.

\section{INQUÉRITO POSTAL - DELPHI TECHNIQUE}

Questionário 1 - Tendo por objetivo, nesta etapa, a obtenção dos parâmetros clínicos para a avaliação do status clínico de atividade ou inatividade/remissão para as ARJ/AIJ/, solicitou-se aos participantes:

1) A lista de sinais e sintomas que deveriam ser considerados na decisão sobre a situação clínica de inatividade ou remissão, de forma aberta (nenhuma lista foi fornecida);

2) informações sobre quanto tempo o paciente deveria estar sem medicação e sem sinais e sintomas indicativos de atividade para ser considerado em remissão;

3) que escrevessem a sua definição de remissão clínica.

Este questionário foi respondido por 130 reumatologistas pediátricos, associados ao PRINTO, ao PRCSG e à Seção de Reumatologia Pediátrica do ACR (Estados Unidos) e Canadá. Os resultados deste primeiro inquérito mostraram a definição de consenso para os seguintes itens:

Um paciente com ARJ/AIJ não pode ser considerado como tendo a doença inativa, se quaisquer dos seguintes itens estiverem presentes:

1. edema articular que não seja devido ao alargamento ósseo;

2. febre atribuída à doença;

3. Rash atribuído à doença;

4. VHS anormal.
Questionário 2 - Este questionário incluiu:

1) Questões do tipo sim/não, como p.ex.: poderia um paciente estar em remissão se os nódulos reumatóides estivessem ainda presentes, mas sem progressão ou aumento em número e tamanho? Resposta Sim / Não

2) Questões abertas, como p.ex.: por quanto tempo deveria estar o paciente com atividade quiescente, na ausência de qualquer medicação para ser considerado clinicamente em remissão?

No questionário 2 foi alcançado o consenso entre os seguintes itens:

Consenso: um paciente com JRA/JIA não pode ser considerado em estado de "doença inativa" se quaisquer dos seguintes itens estiverem presentes:

- serosite;

- uveíte.

Não se obteve consenso nos seguintes itens:

Um paciente com JRA/JIA não pode ser considerado em estado de "doença inativa" se quaisquer dos seguintes itens estiverem presentes:

1. testes laboratoriais anormais estiverem presentes, mas forem definitivamente atribuídos à outra condição clínica que não a artrite;

2. limitação dos movimentos estiver presente, mas for atribuída à seqüela ou dano articular prévio, considerada irreversível e não devida à atividade vigente da artrite;

3. se a dor à palpação estiver presente, mas não for acompanhada de edema, dor à movimentação ou limitação da mobilidade.

\section{Consensus Conference - Nominal Group TECHNIQUE}

A Consensus Conference - utilizando a Nominal Group Technique - é uma reunião estruturada, conduzida por moderadores com reconhecida experiência nesta técnica de discussão, e combinando os resultados da opinião dos especialistas com a análise estatística para a verificação de concordância de pelo menos $80 \%$ entre os participantes.

Vinte reumatologistas pediátricos (listados abaixo) participaram durante dois dias da Consensus Conference em Marco Island, Flórida, EUA, sob a coordenação de três moderadores.

Os seguintes itens alcançaram o consenso com $80 \%$ de concordância entre os especialistas:

Os critérios para doença inativa devem incluir:

1) nenhuma articulação com artrite ativa; 2) ausência de febre, rash, serosites, esplenomegalia ou adenomegalia 
generalizada atribuída à ARJ/AIJ; 3) sem uveíte ativa (critérios de atividade da uveíte a ser definido); 4) VHS ou PCR normal (se ambos forem testados, ambos devem ser normais); 5) a avaliação global de atividade da doença pelo médico deve apresentar o menor valor possível (indicando nenhuma atividade).

O grupo não alcançou consenso nos seguintes critérios:

1) hepatomegalia; 2) nódulos reumatóides; e 3) rigidez matinal.

De acordo com o consenso obtido em reunião plenária, seis meses contínuos de doença inativa são necessários para classificar um paciente como em remissão com medicação; 12 meses contínuos sem medicação e mantendo a doença inativa para ser considerado em remissão sem medicação. O termo "resposta clínica completa" foi considerado inadequado, alcançando $80 \%$ de concordância. O critério final de remissão sem medicação deve prever com acurácia de $95 \%$ que o paciente tem $\leq 20 \%$ de probabilidade de recaída dentro dos próximos cinco anos.
Estes critérios foram desenvolvidos em processo semelhante ao desenvolvido por Pinals et al. (1981) ${ }^{(11)}$ para a artrite reumatóide. Os critérios preliminares aqui apresentados (Tabela 1) têm validade de construção, abrangência e conteúdo. Entretanto, ainda estão em desenvolvimento, devendo ser testados prospectivamente para a acurácia e validade de discriminação, na prática. A validação prospectiva com bancos de dados pré-existentes será seguida pela validação prospectiva em pacientes reais, incluindo todos os subtipos de AIJ.

Entre os tópicos discutidos, os termos AIJ e ARJ foram abordados considerando a maior abrangência dos subtipos definidos na designação das AIJ de acordo com a nomenclatura e classificação da ILAR Task Force ${ }^{(10)}$ e não somente os subgrupos da designação ARJ de acordo com os critérios do $\mathrm{ACR}^{(12)}$. No entanto, os tópicos relativos à entesite, artrite psoriásica e uveítes ainda estão pendentes com questões relativas aos critérios diagnósticos e de atividade, pois mesmo com a opinião de especialistas em uveíte, não se chegou ao consenso sobre atividade/inatividade da uveíte $^{(14)}$.

TABELA 1

DEFINIÇÃO PRELIMINAR ${ }^{(13,14)}$ DOS CRITÉRIOS DE REMISSÃO DA AIJ ${ }^{(A)}$

1. Nenhuma articulação com artrite ativa ${ }^{(b, c)}$

2. Ausência de febre, rash, serosite, esplenomegalia ou adenomegalia generalizada atribuíveis à AIJ

3. Ausência de uveíte ativa, psoríase ou entesite (a ser definida)

4. VHS ou PCR normais (se ambos forem testados, ambos devem ser normais)

5. Avaliação global pelo médico indicando melhor escore possível (de acordo com a escala a ser utilizada) para doença inativa

Avaliações adicionais foram sugeridas, das quais não se obteve consenso, mas poderiam ser incluídas eventualmente na validação prospectiva dos critérios:

- Presença de nódulos reumatóides

- Presença de hepatomegalia

- Presença e duração da rigidez matinal

a. Dois tipos de remissão clínica foram propostos:

- Remissão clínica com medicação. 0 critério para remissão clínica deve ser alcançado por um mínimo de seis meses contínuos enquanto recebendo medicação para ser considerada remissão clínica com medicação.

- Remissão clínica sem medicação. 0 critério para remissão clínica deve ser alcançado por um mínimo de 12 meses contínuos enquanto sem qualquer medicação anti-artrítica para ser considerada remissão clínica sem medicação.

a. AIJ - Definida de acordo com os critérios da ILAR-Task Force $e^{(10)}$

b. $0 \mathrm{ACR}^{(12)}$ define uma articulação ativa como uma articulação com edema que não seja devido ao alargamento ósseo ou, se não houver edema, com limitação dos movimentos acompanhada de dor, calor ou sensibilidade à movimentação.

c. 0 achado isolado de dor à movimentação, sensibilidade ou limitação dos movimentos ao exame articular pode estar presente somente se atribuível ao dano articular prévio (seqüela) em artrite considerada inativa ou devida às causas não reumatológicas como trauma. 
Participaram da Consensus Conference os seguintes membros ${ }^{(13,14)}$.

Organizadores: Nicolino Ruperto, Gênova, Itália; Carol Wallace, Seattle, Washington,EUA, Edward Giannini, Cincinatti, Ohio, EUA.

Participantes: Suzanne Bowyer, Indianápolis, Indiana, EUA; Ruben Cuttica, Buenos Aires, Argentina; Ciaran Duffy, Montreal, Canadá; Flavio Fantini, Milão, Itália; Michael Hofer, Lausanne, Suíça; Norman Ilowite, New Hyde Park, Nova York, EUA; Yukiko Kimura, Hackensack, New Jersey, EUA; Ronald Laxer, Toronto, Canadá; Daniel Lovell, Cincinatti, Ohio, EUA; Claudia Machado, Botucatu, São Paulo, Brasil; Alberto Martini, Gênova, Itália; Kevin Murray, Perth, Austrália; Sheila de Oliveira, Rio de Janeiro, Brasil; Katleen O’Neal, Buffalo, Nova York, EUA; Angelo Ravelli, Gênova, Itália; Lisa Rider, NIH Washington-DC, EUA; Christy Sandborg, Stanford, Califórnia, EUA; Susana Serrate-Sztein, NIAMS Washington-DC, EUA(Observer); David Sherry, Filadélfia, Pensilvânia, EUA; Ruben Burgos-Vargas, Cidade do México, México; Richard Vesely, Kosike, Eslováquia.

\section{PATROCINADORES}

Paediatric Rheumatology International Trials Organization (PRINTO) - European Union - Contract n QLG1-CT2000-00514-EU.

\section{REFERÊNCIAS}

1. French AR, Mason T, Nelson AM, O'Fallon WM, Gabriel SE: Increased mortality in adults with a history of juvenile rheumatoid arthritis: a population-based study. Arthritis Rheum 44: 523-7, 2000.

2. Wallace CA, Levinson JE: Juvenile rheumatoid arthritis: outcome and treatment for the 1990s. Rheum Dis Clin North Am 17: 891-905, 1991

3. Ruperto N, Levinson JE, Ravelli A, et al: Long-term health outcome and quality of life in American and Italian inception cohorts of patients with juvenile rheumatoid arthritis. I-Outcome status. J Rheumatol 24: 945-51, 1997.

4. Guillaume S, Prieur A-M, Coste J, Job-Deslandre C: Long-term outcome and prognosis in oligoarticular-onset juvenile idiopathic arthritis. Arthritis Rheum 44: 1858-65, 2000.

5. Zak M, Pedersen FK: Juvenile chronic arthritis into adulthood: a long term follow up study. Rheumatology 39: 198-204, 2000.

6. Minden K, Niewerth M, Listing J, et al: A Long term outcome in patients with juvenile idiopathic arthritis. Arthritis Rheum 46 2392-401, 2002.

7. Oen K, Malleson PN, Cabral DA, Rosenberg AM, Petty RE, Cheang M: Disease course and outcome of Juvenile Rheumatoid Arthritis in a multicenter cohort. J Rheumatol 29: 1989-9, 2002.
Pediatric Rheumatology Collaborative Study Group (PRCSG), NIH and Children's Hospital of Cincinnati Grant RO3 AI 44046-USA.

Childhood Arthritis \& Rheumatology Research Alliance (CARRA) - USA.

NIAMS, the ACR and Pfizer.

\section{AGRADECIMENTOS}

A colaboração no inquérito junto ao PRINTO: Sheila Knupp Feitosa de Oliveira - Universidade Federal do Rio de Janeiro (UFRJ); Flavio Sztajnbok - Universidade Estadual do Rio de Janeiro (UERJ); Blanca Elena Bica - Universidade Federal do Rio de Janeiro (UFRJ); Maria Odete Esteves Hilário Universidade Federal de São Paulo (Unifesp); Iloite Scheibel - Universidade Federal do Rio Grande do Sul (UFRGS); Virginia Paes Leme Ferriani - Faculdade de Medicina de Ribeirão Preto/Universidade de São Paulo (FMRP-USP); Clovis Artur Almeida Silva - Faculdade de Medicina da Universidade de São Paulo (FMUSP); e Carlos Henrique Martins Silva - UFU-MG.
8. Giannini EH, Ruperto N, Ravelli A, Lovell DJ, Felson DT, Martini A: Preliminary definition of improvement in juvenile arthritis. Arthritis Rheum 40: 1202-9, 1997.

9. Delbecq AL, Van De Ven AH, Gustafson DH (Ed): Group Technique for Program Planning - a Guide to Nominal Group and Delphi processes. Scott, Foresman and Company, Glenview, Illinois, 1975.

10. Petty RE, Southwood TR, Manners P, et al: International League of Associations for Rheumatology classification of juvenile idiopathic arthritis: second revision, Edmonton, 2001. J Rheumatol 31: 390-2, 2004

11. Pinals RS, Masi AT, Larsen RA: Preliminary criteria for clinical remission in rheumatoid arthritis. Arthritis Rheum 24: 1308-15, 1981.

12. JRA Subcommittee of the diagnostic and therapeutic criteria committee of the American Rheumatism Association (ARA); proposed revisions of the JRA criteria. Arthritis Rheum (Suppl.) 20: 195-9, 1977.

13. Proceedings "Consensus Conference to develop criteria for inactive disease in Juvenile Rheumatoid Arthritis" - Marco Island, FloridaUSA. May 03-04, 2003.

14. Wallace CA, Ruperto N, Giannini EH: Preliminary criteria for clinical remission for select categories of Juvenile Idiopathic Arthritis. J Rheumatol 31: 2290-4, 2004. 